\title{
How self-reflection and resilience can affect pre-competition anxiety? Evidence from national competitive table tennis in adolescent players
}

\author{
Lin Wang ${ }^{1,2}$ \\ Accepted: 2 November 2021 \\ (c) The Author(s) 2021
}

\begin{abstract}
Although previous studies have investigated pre-competition anxiety (PCA), the research examining how to promote the development of self-reflection and resilience in order to regulate PCA is limited. This study has three aims: to examine group differences age, gender, sport level, and exercise duration in (PCA), self-reflection and resilience; to test the relationships between PCA, self-reflection, and resilience; and to determine whether self-reflection and resilience can predict PCA. The included 82 male and 105 female adolescent Chinese table tennis player (ages 12-19). SPSS 25.0 software has used to drive ANOVA, Pearson correlations and regression analysis. The main result was that Pearson correlations demonstrated the strongest relationship between PCA, self-reflection, and resilience. The regression analysis model proved that self-insight predicted cognitive anxiety, somatic anxiety and self-confidence; interpersonal assistance and emotional control predicted self-confidence; and emotional control predicted cognitive anxiety and somatic anxiety. The result of this study were consistent with those of previous studies: there was a significant relationship between PCA, self-reflection and resilience when using the Chinese Adolescent Resilience Scale, and there were some differences in the test of group differences. However, this study uncovered new evidence that interpersonal assistance and emotional control can predict PCA. Further research should focus on cross-cultural differences in the process of cultivating athlete self-reflection and resilience.
\end{abstract}

Keywords Pre-competition anxiety(PCA) $\cdot$ Self-reflection $\cdot$ Resilience $\cdot$ Adolescent table tennis players $\cdot$ Regression analysis model

\section{Introduction}

Competitive anxiety, as a common emotion of athletes, has always been a topic of concern in sports science research (Correia \& Rosado, 2019; Rice et al., 2019; Sniras \& Uspuriene, 2018). Anxiety easily leads to attention problems in competitive games (Pena \& Losada, 2017; Pijpers et al., 2005). The distraction of attention not only shows down the athlete's reaction speed and reduces the athlete's ability to predict, but is also affects the rationality of their response to the competition to a certain extent.

Lin Wang

lw679@exeter.ac.uk

1 Guilin University of Electronic Technology, Guilin 541004, China

2 Department of Sport and Health Sciences, College of Life and Environmental Sciences, University of Exeter, Exeter EX1 2LU, UK
Many researchers have discussed the significant relationship between emotion and cognition (Doron \& Martinent, 2017; Martinent \& Ferrand, 2007; Smith et al., 1988), as cognitive development can regulate competitive anxiety (Gomes, 2017). For example, Zhang et al. (2018) directly tested the different cognitive functions that are thought to be impaired when performing under anxiety. Processing efficiency theory states that anxiety creates high cognitive demands, appropriating the resources of the working memory, leading to impaired performance. Previous research supports the experimental evidence (Donachie et al., 2018a; Doron \& Martinent, 2017). Donachie et al. (2018a, b) reported that, perfectionistic cognitions were a positive predictor of anxiety, anger and dejection. Recently, Doron and Martinent (2017) proposed that appraisal can affect anxiety based on the cognitive-motivational-relational theory of emotion because appraisal plays an important role in the anxiety state. When evaluating the significance of an event in relation to the person, individuals' coping strategies can 
be used to mitigate the effect of anxiety on performance (Crane et al., 2019).

Self-reflection is a similar appraisal process that can influence performance based on previous experience (Falon et al., 2021), which if employed it frequently would effectively enhance an athlete's performance by increasing their ability to detect problems and copy strengths (Cowden \& Meyer-Weitz, 2016b). For example, using reflection cards can improve team performance (Hanrahan et al., 2009; Rodriguez-Ayllon et al., 2019). Hanrahan et al. (2009) proposed structured self-reflection is a positive tool that may improve performance and maintain effort and perceived important. Cowden et al. (2016) also demonstrated the effects of selfreflection exercise and self-insight on improving athletic performance.

Moreover, there are some important relationship between self-reflection and appraisal, according to the systematic self-reflection model (Crane et al., 2019). Further, there is evidence that individuals prone to anxiety tend to utilize a conscious and purposeful approach to self-reflection (Grant et al., 2002).

However, few studies focus on the relationship between self-reflection and PCA. Therefore, it is important to investigate how self-reflection influences PCA, and further studies should evaluate others methods that regulate PCA based on empirical evidence. Thus, we hypothesize that there is an important relationship between PCA and self-reflection.

Furthermore, individual differences of athletes may have different effects on their coping with PCA (Rhodes \& Smith, 2006). Some results showed that anxiety was negatively related to resilience in its acceptance dimension (Demir \& Barut, 2020; González-Hernández et al., 2020). Although, resilience, as positive psychological capital, can help people' s growth to a certain extent (Howells et al., 2019; Sarkar $\&$ Fletcher, 2014), there is a light difference in definition, Research over the past 20 years has shown that resilience is a multi-dimensional feature that changes with an athlete's environment, time, age, gender and cultural origin (Pines $\&$ Rutter, 1984). Richardson et al. (1990) proposed a new model of resilience, starting from a point in the bio-psychospiritual balance (homeostasis), in which one adapts the body, mind and spirit to the current living environment. Internal and external stressors are always present, and one's ability to cope with these events is affected by disturbances before successful and failed adaptations (Connor \& Davidson, 2003).

Specially, in China, this concept overlaps with the 'adversity view' in traditional Chinese culture. Under the influence of dialectical thinking, Chinese people hold an optimistic and dialectical attitude towards adversity and misfortune, believing that adversity is 'a great task that has come from heaven' and 'a blessing in disguise'. Such as the attitudes that "adversity makes a person talented man grow", (Hu \& Gan, 2008a), people's growth cannot ignore the help and encouragement of others, especially children and adolescent. However, $\mathrm{Hu}$ and Gan (2008a) conducted a systematic review of previous psychological resilience questionnaires, mainly because the varying dimensions are assessed by different psychological resilience scales. These cultural features focus resilience at its core on people's own abilities. For example, Wagnild and Young (1993) resilience scale used two factors, including personal energy and acceptance of self and life. Connor and Davidson (2003) compiled the CD-Resilience Scale that includes five factors, namely ability, tolerance of negative emotions, acceptance of change, sense of control, and spiritual beliefs.

Therefore, in terms of literature review, we assume that interpersonal assistance and emotional control in resilience are more effective than other factors for mitigating PCA in adolescent athletes in China, the adolescence resilience scale (Hu \& Gan, 2008a) is suitable to our study, which focused on the "process" of adolescents encountering adversity and significant pressure. It also identified what factors have positive effects on the healthy development of adolescents. Lastly, the five dimensions of the scale include goal focus, emotional control, positive cognition, family support, and interpersonal support.

Moreover, the systematic self-reflection (SSR) model explains the reflective process as a series of outcomes that, promote the capacity for resilience and, change with time and opportunities (Crane et al., 2019). Meanwhile, according to Cowden and Meyer-Weitz (2016a), a multivariate regression analysis revealed that self-insight was the only significant predictor of both resilience and stress. However, their study does not address the. Such as differences between sport level groups. Based on social cognitive theory, age, gender differences can influence the emotions, cognition and individuality of adolescents and children. Therefore, we hypothesis that there is a significant heterogeneity of group differences in PCA, self-reflection and resilience.

Although previous studies have attempted to explain the relationship between self-reflection and resilience in competitions (Cowden and Meyer-Weitz, 2016a, b), the importance of PCA in athletes' performance has not been considered. Therefore, this study aimed to explore how to promote the development of self-reflection and resilience in order to regulate PCA, and the present study has several aims to achieve this goal. The present study has several aims:1) to examine the relationship between PCA, self-reflection and resilience; 2) to explore the important factors of self-reflection and resilience, which can affect PCA in adolescent table tennis athletes in China; 3 ) to found the heterogeneity based on 
different sport level groups, age, gender in PCA, self-reflection and resilience.

\section{Methodology}

\section{Research Design}

We designed a cross-sectional study using a quantitative data collection method. We used convenience sample, and baseline characteristics (e.g., gender, age, sport level, and exercise duration) were collected via surveys. Measures for self-reflection, PCA and resilience were collected using psychological scales. All data collection began 48 before the competition and was completed before the game.

\section{Participants}

A total of 206 athletes ranging in age from 12 to 19 years (mean age $=18.6$ years; \pm 1.8 ), convenience selected from 750 young athletes participating in the 20th China National Junior High School Table Tennis Championships. One-hundred and eighty-seven valid questionnaires (82 male and 105 female) were recovered, and the effective rate was $90.78 \%$. Participants gave written, informed consent to participate in the study. All data in this study were collected in accordance with the ethical standards of the Helsinki Declaration.

\section{Procedure}

This study investigated the application of psychological questionnaires to measure the PCA, self-reflection and resilience of younger in the national youth table tennis competition to explain how self-reflection and resilience affect PCA. The investigators received permission from the event organizing committee before the game to freely enter and exit the competition venue and contact various sports teams. When each team reported to the competition organizing committee, a researcher explained the purpose of the study to the team leader or coach, seeking their consent. The investigators had a certain understanding of the competition time, arrangements and basic situations of the participants before the game, and actively communicated with the team's leading teacher and coaches to explain the research purpose and content, which caused better responses.

If the coach is willing to be investigated, the investigator can go to the field to randomly survey the athletes during the adaptation stage, or go to the athletes lounge to conduct surveys of the athletes. The measurement time was $48 \mathrm{~h}$ before the competition. The research-related information was transmitted to the test subjects before the measurement. The authors met the participants and informed them that the researchers were interested in understanding athletes' anxiety, resilience and self-reflection during sports. After the investigators explained the study, the athletes were free to choose whether to remain anonymous. The athletes were cooperative and took the questionnaire seriously.

\section{Measure}

PCA was measured using the Chinese State Anxiety Scale (CSAI-2) revised by Zhu (1994), which is commonly used to investigate PCA in China. The questionnaire consisted of three subscales of cognitive anxiety, somatic anxiety and self-confidence. Each subscale had nine questions for a total of 27 questions. The revised China Norm Scale uses internal consistency to determine the reliability of the scale. The results show that the three-step two-step internal rating consistency is between 0.86 and 0.72 . The validity of the scale is mainly based on the coexistence validity, the independence of each subscale and the predicted validity, and the test results have good validity (Zhu, 1994).

The Self-Reflection and Insight Scale (Grant et al., 2002) was employed to measure the level of self-reflection in the adolescent athletes, which has a version translated into Chinese. Before administering the pre-competition survey, the scale was also tested for reliability and validity, and the parameters met the parameters met the survey requirements. The scale is one of the tools for measuring individual selfreflection and insight, including two governors. One factor is called self-reflection (SRIS-SR), and the other is insight. According to Grant et al. (2002), the result of the factor loadings and the coefficient alpha for the self-reflection scale was .91 and .87 for the insight scale. The scale uses a retest reliability method with intervals of seven weeks, which was .77 (SRIS-SR) and .78 (SRIS-IN).

The Adolescent Psychological Resilience Scale (Hu \& Gan, 2008a) has traditional Chinese characteristics based on the process theory of psychological resilience. This scale was developed based on the characteristics of Chinese adolescents, and the scale structure and items are suitable for this study. The questionnaire includes 27 questions looking at five factors: emotional control, positive cognition, interpersonal assistance, goal focus and family support. Their coefficient alpha was under 0.7 . The five factors of the scale are more closely related to the topics discussed in this study, due to the study being aimed at the young athlete group; therefore, this scale is appropriate.

\section{Statistical Analysis}

The statistical analysis followed the procedures outlined in the Statistical Package for Social Sciences (SPSS, Version 25.0). First, the sample are grouped according to the baseline 
of the adolescent athletes, gender (boys and girls) and age ( 2 years is the cut-off point), exercise duration $(1-4 ; 5-9$; and above or equal 9), and sport levels (national level 1, national level 2 and without level). Athletes are divided into master class, national level 1, national level 2, and without level based on the grading standards of the General Administration of Sport of China. First, we used ANOVA to determine whether different factors (age, gender, exercise duration and sport level) influenced the independent variables PCA, self-reflection and resilience). We also hypothesized that homogeneity of variance of the independent variables, which are continuous variables subject to normal distrution, would not be significant.

Second, we used Pearson correlations (one-tailed) to determine whether significant relationships exist between PCA, self-reflection, and resilience in adolescent table tennis athletes. We also hypothesized that there is a significant relationship.

Finally, after proving that PCA, resilience and self-reflection are relevant, we sought to discover if there is a causal relationship between the dimensions of the three variables. We used cognitive and somatic PCA as the dependent variables and reflect involved, reflection needs, and insight of self-reflection, as independent variables. Furthermore, the five factors of resilience, were used as independent variables, in a regression analysis model to determine whether selfreflection, self-insight and resilience predict PCA.

\section{Result}

One-hundred and eighty-seven people provided sufficient information from the baseline questionnaire, which includes gender, age, sport level and exercise duration, on how to influence PCA, resilience and self-reflection among young athletes. ANOVA had been used to find the effect size of demographic variables when resilience and self-reflection were the dependent variables, since a one-way analysis of variance can test whether a single factor affects one or several independent dependent variables and whether the differences between different levels of groupings are statistically significant. Self-reflection was the dependent variable to explore the influence of demographics, where the process employs the ANOVA. Due to multivariate analyses of variance, multiple factors cause changes in the dependent variable and the impact of such factors may be a single factor or a combination of multiple factors. The Pearson product-moment correlation coefficient was employed to find bivariate correlations for PCA, resilience and self-reflection (Donachie et al., 2018a, b) (Table 1).

All the variables satisfied the tests of homoskedasticity (Levene's homogeneity test) in terms of the variables of the Levene's test $\geq 0.05$; therefore, the ANOVA could be investigated in PCA of the adolescent athletes. Even though much research has indicated that there are some significant differences between male and female athletes in competition anxiety, the results of the data analysis showed that differences
Table 1 Scales used to measure variables and description of the sample in PCA $(n=187)$

\begin{tabular}{|c|c|c|c|c|c|}
\hline \multirow[b]{2}{*}{ Variables } & \multirow[b]{2}{*}{$n$} & \multicolumn{4}{|c|}{ Pre-competition Anxiety } \\
\hline & & Anxiety M (SD) & $P$ & Self-confidence M (SD) & $P$ \\
\hline Gender & & $34.02(9.49)$ & 0.255 & $23.64(5.22)$ & $0.006 * *$ \\
\hline Male & 82 & $33.06(9.32)$ & & $24.79(5.08)$ & \\
\hline Female & 105 & $34.76(9.6)$ & & $22.74(4.98)$ & \\
\hline Age & & $34.02(9.49)$ & 0.227 & $23.64(5.11)$ & $0.013^{*}$ \\
\hline $12-13$ & 23 & $35.74(7.44)$ & & $22.48(5.38)$ & \\
\hline $14-15$ & 56 & $32.38(9.08)$ & & $25.13(4.67)$ & \\
\hline $16-17$ & 82 & $35.16(10.28)$ & & $23.00(5.04)$ & \\
\hline $18-19$ & 26 & $32.38(9.04)$ & & $24.38(5.21)$ & \\
\hline Level & & $34.02(9.49)$ & 0.423 & $23.64(5.11)$ & $0.034^{*}$ \\
\hline Nation 1 & 65 & $32.83(8.82)$ & & $24.95(5.09)$ & \\
\hline Nation 2 & 97 & $34.46(10.14)$ & & 23.03 (4.97) & \\
\hline None & 25 & $35.36(8.52)$ & & $22.60(5.25)$ & \\
\hline Years & & $34.02(9.49)$ & & $23.64(5.11)$ & \\
\hline $1-4$ & 18 & $37.50(7.20)$ & & $22.33(5.04)$ & \\
\hline $5-8$ & 99 & $33.77(10.06)$ & & $23.29(5.18)$ & \\
\hline$\geq 9$ & 70 & $33.47(9.49)$ & & $24.47(4.97)$ & \\
\hline
\end{tabular}

$\mathrm{M}=$ mean; $\mathrm{SD}=$ standard deviation

$* p<0.05$. $* * p<0.01$ 
in competition anxiety between male and female athletes are not statistically significant in table tennis adolescent athletes.

Additionally, age, performance level and training years are also without an important effect on cognitive and

Table 2 Scales used to measure variables and description of the sample resilience $(n=187)$

\begin{tabular}{lllll}
\hline & & $l$ & \\
\cline { 3 - 4 } Variables & $n$ & M (SD) & & \\
\hline Gender & & $98.16(14.71)$ & 0.812 & 0.128 \\
Male & 82 & $100.01(14.71)$ & & \\
Female & 105 & $96.70(14.62)$ & & \\
Age & & $98.16(14.71)$ & 0.708 & 0.069 \\
12-13 & 23 & $97.13(13.69)$ & & \\
14-15 & 56 & $99.21(17.45)$ & & \\
16-17 & 82 & $96.41(13.29)$ & & \\
$18-19$ & 26 & $102.27(13.15)$ & & \\
Level & & $14.719(98.16)$ & 0.116 & 0.494 \\
Nation 1 & 65 & $100.69(13.49)$ & & \\
Nation 2 & 97 & $97.59(15.63)$ & & \\
None & 25 & $93.76(13.32)$ & & \\
Years & & $98.16(14.71)$ & 0.106 & 0.218 \\
$1-4$ & 18 & $93.44(11.50)$ & & \\
$5-8$ & 99 & $99.62(16.30)$ & & \\
$\geq 9$ & 70 & $97.30(12.81)$ & & \\
\hline
\end{tabular}

$\mathrm{M}=$ mean; $\mathrm{SD}=$ standard deviation

$* p<0.005 . * * p<0.001$ somatic anxiety, but the self-confidence of adolescent athletes in the national competition had by gender $(p=0.006)$, age $(p=0.013)$ and sport level $(p=0.034)$. The results found that male players definitely had a greater tendency towards expressing their self-confidence, players of 14-15 years had exposed much self-confidence and a high level like the nation 1 of athlete always had the high score in their selfconfidence (Table 2).

All the variables satisfied the tests of homoskedasticity (Levene's homogeneity test) in terms of the variables of Levene's test $\geq 0.05$; therefore, the ANOVA could be investigated in the resilience of adolescent athletes. The above data results show that various factors in demography have no significant impact on young table tennis players.

All the variables satisfied the tests of homoskedasticity (Levene's homogeneity test) in terms of the variables of Levene's test $\geq 0.05$; therefore, the ANOVA could be investigated in the self-reflection of the adolescent athlete. According to the data of Table 3, the insight factor of adolescent table tennis athletes had an effect $(p=0.03)$ on gender, and males had higher insight than females. Simultaneously, in terms of training years of adolescent table tennis player had statistical findings in the scores of total self-reflection and the self-reflection factor $(p=0.012, p=0.002)$. Again, the adolescent athletes with five to eight training years wanted to engage in self-reflection, the self-reflection score was higher than the player with nine training years, but their insight factor score was obviously lower (Table 4).
Table 3 Scales used to measure variables and description of the sample in self-reflection $(\mathrm{n}=187)$

\begin{tabular}{|c|c|c|c|c|c|c|c|}
\hline \multirow[b]{2}{*}{ Variables } & \multirow[b]{2}{*}{$n$} & \multicolumn{2}{|c|}{ Self-reflection (T) } & \multicolumn{2}{|c|}{ Self-reflection (R) } & \multicolumn{2}{|c|}{ Self-reflection (I) } \\
\hline & & $\mathrm{M}(\mathrm{SD})$ & $p$ & $\mathrm{M}(\mathrm{SD})$ & $p$ & $\mathrm{M}(\mathrm{SD})$ & $p$ \\
\hline Gender & & $72.35(8.31)$ & 0.416 & $45.30(5.68)$ & 0.570 & & $0.03 *$ \\
\hline Male & 82 & $72.91(8.74)$ & & $45.04(5.99)$ & & $27.88(4.50)$ & \\
\hline Female & 105 & 71.91 (7.97) & & $45.51(5.45)$ & & $26.40(4.66)$ & \\
\hline Age & & $72.35(8.31)$ & 0.122 & $45.30(5.68)$ & 0.367 & & 0.130 \\
\hline $12-13$ & 23 & $70.22(8.81)$ & & $44.52(6.28)$ & & $25.70(4.73)$ & \\
\hline $14-15$ & 56 & $73.21(9.49)$ & & $46.04(6.05)$ & & $27.18(5.43)$ & \\
\hline $16-17$ & 82 & $71.50(7.61)$ & & $44.70(5.35)$ & & $26.80(4.14)$ & \\
\hline $18-19$ & 26 & $75.08(6.62)$ & & $46.35(5.30)$ & & $28.73(3.87)$ & \\
\hline Level & & $95.32(10.89)$ & 0.535 & $45.30(5.68)$ & 0.425 & & 0.447 \\
\hline Nation 1 & 65 & $96.08(10.75)$ & & $45.57(5.57)$ & & $27.63(4.34)$ & \\
\hline Nation 2 & 97 & $95.36(10.29)$ & & $45.48(5.29)$ & & $26.69(4.76)$ & \\
\hline None & 25 & $93.20(13.45)$ & & $43.92(7.92)$ & & $26.92(4.93)$ & \\
\hline Years & & $95.32(10.89)$ & $0.012 * *$ & $45.30(5.68)$ & $0.002 * *$ & & 0.175 \\
\hline $1-4$ & 18 & $90.17(13.45)$ & & $42.67(6.92)$ & & $25.22(4.51)$ & \\
\hline $5-8$ & 99 & $97.32(10.23)$ & & $46.63(5.32)$ & & $27.05(4.87)$ & \\
\hline$\geq 9$ & 70 & $93.81(10.56)$ & & $44.11(5.40)$ & & $27.51(4.25)$ & \\
\hline
\end{tabular}

Self-reflection (T) is the total score of self-reflection in adolescent athletes, self-reflection (R) is the dimensionality of reflection and self-reflection (I) is the insight factor. $\mathrm{M}=$ mean; $\mathrm{SD}=$ standard deviation $* p<0.05 . * * p<0.01$ 


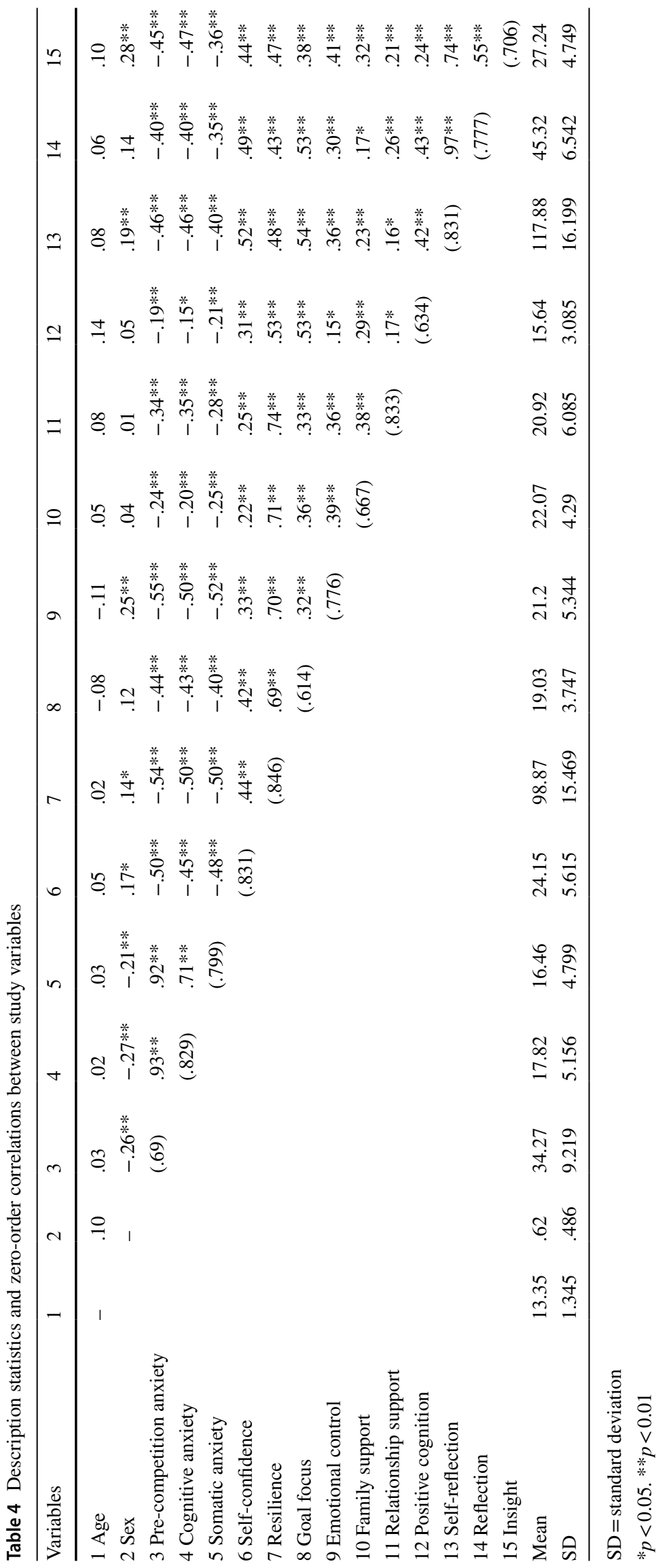


Based on correlational analyses using Cohen's recommendation (1988), the results of the relationship between PCA and psychological resilience showed that cognitive anxiety and goal resilience in mental resilience was significantly negatively related to emotional control and interpersonal support. Physical anxiety was significantly negatively related to emotional control. Self-confidence had a significant positive correlation with goal concentration, emotional control, family support and interpersonal support. It is worth noting that the relationship between the dimensions of self-reflection and PCA was manifested only in the significant negative correlation between insight and cognitive anxiety and in the positive correlation between self-reflection, insight dimensions and self-confidence. According to the relationship between self-reflection and resilience, a crucial finding between the factors of self-reflection, insight and emotion control had more of an effect on the statistics. Positive cognition also had a significant influence on the self-reflection and insight of the athletes. Meanwhile, the insight factor of self-reflection had been affected by family and relationship support of resilience.

In order to further explore the relationship between PCA and the resilience of adolescent table tennis players based on the above research, we performed a stepwise regression analysis of various factors. Using the factors of resilience as independent variables, cognitive anxiety, somatic anxiety and self-confidence of PCA was the dependent variable. The analysis results are shown in the following Table 5, emotional control and interpersonal assistance predicted the self-confidence of pre-competition anxiety, explaining $14.9 \%$ and $18.7 \%$ of the variance in self-confidence. Further, emotional control predicted cognitive anxiety and somatic anxiety.

The self-reflection factors were used as predictor variables, and PCA was used as the dependent variable. A stepwise regression analysis was used to explain how selfreflection influenced PCA. The results (see Table 6) indicated that self-insight predicted PCA dimensions: $12.8 \%$ of the variance in cognitive anxiety and $8.4 \%$ of the variance in somatic anxiety. Additionally, self-insight also predicted self-confidence; self-insight constructed $8.4 \%$ of the variance in self-confidence.

\section{Discussion}

The purpose of this study was to determine which factors influence PCA. The study's main finding was that there is a significant relationship between PCA, self-reflection and resilience. Self-reflection and resilience have a negative relationship with PCA. There is a cognitive relationship between self-reflection and resilience in adolescent table tennis athletes.

The results demonstrate important heterogeneous differences in PCA in terms of age, gender, sport level. Regarding heterogeneous gender differences, our results differed compared to previous studies in self-reflection. Age, sport level and exercise duration are also without an important effect on cognitive and somatic anxiety, but the
Table 5 Regression model of the interaction between PCA and resilience
Table 6 Regression model of the interaction between PCA and self-reflection

\begin{tabular}{lllllll}
\hline Model & Multiple $\mathrm{R}$ & $\mathrm{R}^{2}$ & Adjusted $\mathrm{R}^{2}$ & $\mathrm{~F}$ & P value & Beta \\
\hline Self-confidence & & & & & & \\
Emotional control & .38 & .14 & .14 & 32.36 & $.00^{* *}$ & .375 \\
Interpersonal assistance & .43 & .18 & .17 & 21.16 & $.004^{* *}$ & .211 \\
Cognitive anxiety & & & & & & \\
Emotional control & .47 & .22 & .22 & 54.86 & $.00^{* *}$ & -.478 \\
Somatic anxiety & & & & & & \\
Emotional control & .38 & .14 & .14 & 31.27 & $.00^{* *}$ & -.315 \\
\hline
\end{tabular}

$* p<0.05 . * * p<0.01$

\begin{tabular}{lllllll}
\hline Model & Multiple $\mathrm{R}$ & $\mathrm{R}^{2}$ & ${\text { Adjusted } \mathrm{R}^{2}}$ & $\mathrm{~F}$ & P value & Beta \\
\hline $\begin{array}{l}\text { Self-confidence } \\
\text { Self-insight }\end{array}$ & .293 & .086 & .081 & 17.399 & $.00^{* *}$ & -.293 \\
$\begin{array}{l}\text { Cognitive anxiety } \\
\text { Self-insight }\end{array}$ & .358 & .128 & .124 & 27.260 & $.00^{* *}$ & -.358 \\
$\begin{array}{l}\text { Somatic anxiety } \\
\text { Self-insight }\end{array}$ & .290 & .084 & .079 & 17.016 & $.00^{* *}$ & 290 \\
\hline
\end{tabular}

$* p<0.05 . * * p<0.01$ 
self-confidence of adolescent athletes in the national competition had significant differences in gender $(p=0.006)$ and, age $(p=0.013)$ and sport level $(p=0.034)$. Males had greater insight than females in adolescent athletes.

Moreover, in the regression model with PCA as the dependent variables, the strong mechanism connections are interpersonal assistance, and emotional control of resilience. These results fit our hypothesis. Insight entered the regression model when PCA was the dependent variable and self-reflection was the independent variables. Therefore, insight is more likely to be an important mechanism for athletes to regulate PCA through self-reflection.

\section{Self-Reflection}

Although there is some evidence of a significant relationship between anxiety and self-reflection in nurses' work experience (Ganzer \& Zauderer, 2013), this study offers strong evidence in the context of athletic competition. This is the first empirical data demonstrating the important relationship between PCA and self-reflection, insight plays a key role in influencing PCA. An explanation for the link between insight, emotional control and anxiety may be that, people with greater insight are more likely to be emotionally stable and less likely to be disturbed by external factors (Nakajima et al., 2019). Such people have better problem-solving skill, which makes them more self-confident, thereby reducing the impact of anxiety under stress (Hagan et al., 2017). Additionally, Herwig (2012) proves that the self-reflection process is closely related to the brain mechanism of the cognitive process. Self-reflection, compared with reflecting on the other persons and to a major part also compared with perceiving photographs of one-self, was associated with more prominent dorsomedial and lateral prefrontal, insular, anterior and posterior cingulate activations (Herwig et al., 2012). And the brain mechanism of the anxiety process that is associated with anxiety related to bilateral insula activation, and worry related to medial prefrontal cortex activation (Knight et al., 2019).

The result of this study found that is males had greater insight than females in adolescent athletes; there was important differences in exercise duration in factors of selfreflection (reflection need and reflection engage). However, there was no significant differences in age, sport level and exercise duration. Self-reflection ability of females' athletes is lower than males, the biology and cognitive ability may be the important factors. Therefore, female athletes need to improve their ability of find problems. Furthermore, the exercise duration affects the level of reflection, without insight, which indicates that the longer the training of athletes, the stronger the motivation of reflection, but it does not mean that athletes with high reflective motivation can touch high ability to find and solve problems in the sports. Further, athletes and coaches should be considering about how to make reflection more effective and improve insight ability.

Developing athletes' reflective ability would help them manage PCA. However, there is limited evidence on effective methods to develop athletes' self-reflective abilities, especially self-insight. In order to regulate the anxiety of athletes through the improvement of their insights, the coach need to stimulate the need for athletes to reflect on their own participation, because self-reflection is a prerequisite for self-insight (Sallis et al., 2000). After the survey of the national youth table tennis players, some athletes developed a reflective habit. Specially, younger athletes and athletes with lower competitive levels have less self-reflection, and coaches have also played an important role in promoting athletes' self-reflection, which is important in self-regulation (Cleary \& Zimmerman, 2001). Thus, coaches should pay attention to the stimulation and cultivation of athletes' selfreflection needs at different strategies, and help athletes find their strengths and weaknesses. This is more targeted and can promote the development of athletes in a more specific way. Coaches use self-motivated beliefs when inspiring athletes to reflect on the need for participation. Self-motivation plays an important role in the goals set by athletes and their game planning (Collins \& Durand-Bush, 2014), which is a crucial channel to facilitate the participation of self-reflection in an athlete when they have planned their goal definitely. In other words, if they would like to achieve success, they probably prefer to find some clue from their experience of competition and training.

Self-insight is more likely to perceive that they will achieve their goals, improve their abilities and have a positive sense of the future. Optimistic goal-setting and outlooks for the future will have an impact on motivation and determination for achievement. In the process of achieving their goal, they will reflect and summaries, adjust imperfect training methods, attitudes and emotions, and apply the process that is beneficial to the realization of goals for future training and competition.

Specifically, the process of developing self-reflection may enhance people's insight, and this, in turn, may affect people's ability to regulate emotions (Crane et al., 2019). Indeed, as people's ability to detect and solve problems improves, they may also be able to predict future problems and prepare solutions in advance, which will reduce their levels of anxiety.

\section{Resilience}

Previous research evidence shows that there is a significant relationship between resilience and competition anxiety (Sarkar and Fletcher, 2014; Zurita-Ortega et al., 2018). This study adopted different measure tools based on Chinese culture and touch the same results (González-Hernández et al., 
2020; Machida et al., 2017). Moreover, according to the regression results, interpersonal assistance and emotional control are the main factors affecting the self-confidence. Emotional control is the only factor that affects athletes' cognitive anxiety, and emotional control and interpersonal assistance can alleviate somatic anxiety.

Emotional control is a special factor in this study's measurement tool. 'emotional control' refers to controlling and adjusting mood swings and pessimism during difficult times. According to $\mathrm{Hu} \& \mathrm{Gan}$ (2008b), these differences may reflect unique features of process-based resilience, or may be unique components of Chinese adolescents' resilience. As described in the introduction, Confucian beliefs about' restraining emotional expression. Taoist advocacy of peace of mind, Chinese people's dialectic thoughts and collectivist coping strategies when deal with adversity may be reflected in their coping processes specifically in terms of emotional control. However, the cross-cultural impact of this study is unclear, and it may be necessary to investigate different groups in the further.

Additionally, interpersonal assistance mostly comes from the sum of factors such as coaches and teammates, and the supportive role of coaches for athletes occupies an irreplaceable position (Kegelaers \& Wylleman, 2018). The relationship between the athletes and the effectiveness of the coach's interpersonal support has become another impact factors affecting the athletes' abilities to regulate and control anxiety (Kegelaers \& Wylleman, 2018). Because of providing positive feedback is a type of attribute affirmation. Attribute affirmation can be implemented by giving participants positive feedback on their personality or by asking participants to list their positive aspects or experiences. In short, both value affirmation and attribute affirmation will restore or enhance one's sense of self-integrity (self-worth). After self-affirmation, people will perceive a higher overall sense of self-holiness, making them stronger and reducing their defensive response to threats (Yang et al., 2015).

Positive coach-to-athlete relationships and constructive coaching behaviour help athletes overcome challenges and increase their ability to overcome setbacks. Davis et al., (2019) research findings focused on direct effects and found that good relationships characterized by trust, commitment and cooperation create a social environment in which athletes are more likely to communicate. Therefore, relationship quality and communication affect each other, and this interconnection may be an important factor affecting athletes' performances and psychological characteristics. Therefore, coaches should attach importance to the establishment of supportive relationships with athletes and build trust and open cooperative relations based on good communication.

\section{Contribution and Limitation}

This study not only demonstrated the relationship between PCA, self-reflection and resilience, but also identified several important factors that greatly impact athletes' ability to regulate PCA, such as insight, emotional control and interpersonal assistance. Moreover, we examined the heterogeneity of PCA, self-reflection and resilience in gender, age and sport level groups. This paper also utilises concepts specific to Chinese culture. For example, the adolescent resilience scale focuses on the development process in relation to adversity experienced in adolescence because Chinese culture believes that individuals can grow when they confront adversity in their lives. Additionally, this follow-up study provides favorable empirical evidence for understanding group differences. In addition, the study data is based on the survey the 20th China National Junior High School Table Tennis Championships, which is very representative. However, this study has some limitations. First, resilience questionnaire is designed based on Chinese adolescents and its use in other cultural environments in related fields, may yield different results. Such as an important factor in resilience is interpersonal support, which can influence the anxiety levels of adolescent athletes.

In other words, cultural differences may affect the universality of the measurement tool's results. Future research could examine cultural differences and propose different strategies to improve resilience in athletes from different cultures. Second, the sample only included table tennis players. As differences in the sports group can affect the regulation effect of reflection and resilience on PCA, further studies could explore whether differences in sports events have an impact on the three variables.

\footnotetext{
Submission of an article implies that the work described has not been published previously (except in the form of an abstract or as part of a published lecture or academic thesis), that it is not under consideration for publication elsewhere, that its publication is approved by all authors and tacitly or explicitly by the responsible authorities where the work was carried out, and that, if accepted, it will not be published elsewhere in the same form, in English or in any other language, including electronically without the written consent of the copyrightholder.
}

Funding China Scholarship Council, Grant/Award Numbers:202108450008

Data Availability The datasets generated during and/or analysed during the current study are available after publication if required by the journal. 


\section{Declarations}

Ethical This study will follow the COPE guidelines on how to deal with potential acts of misconduct. Ethics Committee approval was obtained from the Institutional Ethics Committee of Hunan University of science and technology to the commencement of the study.

Open Access This article is licensed under a Creative Commons Attribution 4.0 International License, which permits use, sharing, adaptation, distribution and reproduction in any medium or format, as long as you give appropriate credit to the original author(s) and the source, provide a link to the Creative Commons licence, and indicate if changes were made. The images or other third party material in this article are included in the article's Creative Commons licence, unless indicated otherwise in a credit line to the material. If material is not included in the article's Creative Commons licence and your intended use is not permitted by statutory regulation or exceeds the permitted use, you will need to obtain permission directly from the copyright holder. To view a copy of this licence, visit http://creativecommons.org/licenses/by/4.0/.

\section{References}

Cleary, T. J., \& Zimmerman, B. J. (2001). Self-regulation differences during athletic practice by experts, non-experts, and novices. Journal of Applied Sport Psychology, 13(2), 185-206.

Collins, J., \& Durand-Bush, N. (2014). Strategies used by an elite curling coach to nurture athletes' self-regulation: A single case study. Journal of Applied Sport Psychology, 26(2), 211-224. https://doi. org/10.1080/10413200.2013.819823

Connor, K. M., \& Davidson, J. R. T. (2003). Development of a new resilience scale: The Connor-Davidson resilience scale $(\mathrm{CD}$ RISC). Depression and Anxiety, 18(2), 76-82. https://doi.org/10. 1002/da.10113

Correia, M. E., \& Rosado, A. (2019). Anxiety in athletes: Gender and type of sport differences. International Journal of Psychological Research, 12(1), 9-17. https://doi.org/10.21500/20112084.3552

Cowden, R. G., \& Meyer-Weitz, A. (2016a). Self-reflection and selfinsight predict resilience and stress in competitive tennis. Social Behavior and Personality, 44(7), 1133-1149. https://doi.org/10. 2224/sbp.2016.44.7.1133

Cowden, R., \& Meyer-Weitz, A. (2016b). Sports mental toughness questionnaire: Evaluation for use in south African competitive tennis. Journal of Sport Behavior, 39(4), 372.

Cowden, R. G., Meyer-Weitz, A., \& Oppong Asante, K. (2016). Mental toughness in competitive tennis: relationships with resilience and stress. Frontiers in Psychology, 7, 320.

Crane, M. F., Searle, B. J., Kangas, M., \& Nwiran, Y. (2019). How resilience is strengthened by exposure to stressors: The systematic self-reflection model of resilience strengthening. Anxiety, Stress and Coping, 32(1), 1-17. https://doi.org/10.1080/10615806.2018. 1506640

Davis, L., Jowett, S., \& Tafvelin, S. (2019). Communication strategies: The fuel for quality coach-athlete relationships and athlete satisfaction. Frontiers in Psychology, 10, 2156.

Demir, A., \& Barut, A. I. (2020). The relationship between university students' psychological resilience and anxiety levels and comparison in terms of physical activity levels gender and academic achievement. Baltic Journal of Health and Physical Activity, 12(1), 75-83. https://doi.org/10.29359/BJHPA.2020.Suppl.1.08

Donachie, T. C., Hill, A. P., \& Hall, H. K. (2018a). The relationship between multidimensional perfectionism and pre-competition emotions of youth footballers. Psychology of Sport and Exercise,
37(February 2019), 33-42. https://doi.org/10.1016/j.psychsport. 2018.04.002

Donachie, T. C., Hill, A. P., \& Hall, H. K. (2018b). The relationship between multidimensional perfectionism and pre-competition emotions of youth footballers. Psychology of Sport and Exercise, 37(March), 33-42. https://doi.org/10.1016/j.psychsport.2018.04. 002

Doron, J., \& Martinent, G. (2017). Appraisal, coping, emotion, and performance during elite fencing matches: A random coefficient regression model approach. Scandinavian Journal of Medicine and Science in Sports, 27(9), 1015-1025. https://doi.org/10.1111/ sms. 12711

Falon, S. L., Karin, E., Boga, D., Gucciardi, D. F., Griffin, B., \& Crane, M. F. (2021). A clustered-randomized controlled trial of a selfreflection resilience-strengthening intervention and novel mediators. Journal of Occupational Health Psychology, 26(1), 1-19. https://doi.org/10.1037/ocp0000268

Ganzer, C. A., \& Zauderer, C. (2013). Structured learning and selfreflection: Strategies to decrease anxiety in the psychiatric mental health clinical nursing experience. Nursing Education Perspectives, 34(4), 244-247. https://doi.org/10.5480/1536-5026-34.4. 244

Gomes, A. R. (2017). Adaptação humana ao stress em contextos desportivos: Teoria, avaliação, investigação e intervenção. In Motricidade (Vol. 13, Issue 1, pp. 3-18). https://doi.org/10.6063/ motricidade.7697.

González-Hernández, J., Gomariz-Gea, M., Valero-Valenzuela, A., \& Gómez-López, M. (2020). Resilient resources in youth athletes and their relationship with anxiety in different team sports. International Journal of Environmental Research and Public Health, 17(15), 1-11. https://doi.org/10.3390/ijerph17155569

Grant, A. M., Franklin, J., \& Langford, P. (2002). The self-reflection and insight scale: A new measure of private self-consciousness. Social Behavior and Personality, 30(8), 821-836. https://doi.org/ 10.2224/sbp.2002.30.8.821

Hagan, J. E., Pollmann, D., \& Schack, T. (2017). Elite athletes' inevent competitive anxiety responses and psychological skills usage under differing conditions. Frontiers in Psychology, 8(DEC), 1-11. https://doi.org/10.3389/fpsyg.2017.02280

Hanrahan, S. J., Pedro, R., \& Cerin, E. (2009). Structured self-reflection as a tool to enhance perceived performance and maintain effort in adult recreational salsa dancers. Sport Psychologist, 23(2), 151-169. https://doi.org/10.1123/tsp.23.2.151

Herwig, U., Kaffenberger, T., Schell, C., Jäncke, L., \& Brühl, A. B. (2012). Neural activity associated with self-reflection. BMC Neuroscience, 13(1). https://doi.org/10.1186/1471-2202-13-52

Howells, K., Sivaratnam, C., May, T., Lindor, E., McGillivray, J., \& Rinehart, N. (2019). Efficacy of group-based organised physical activity participation for social outcomes in children with autism spectrum disorder: A systematic review and Meta-analysis. Journal of Autism and Developmental Disorders, 49(8), 3290-3308. https://doi.org/10.1007/s10803-019-04050-9

Hu, Y., \& Gan, Y. (2008a). Development and validation of adolescent resilience scale. Journal of Psychology, 40(8), 902-912.

$\mathrm{Hu}$, Y., \& Gan, Y. (2008b). Development and validity verification of the adolescent mental resilience scale. Chinese Journal of Psychology, (8), 902-912. http://journal.psych.ac.cn/acps/EN/Y2008/ V40/I08/902.

Kegelaers, J., \& Wylleman, P. (2018). Exploring the Coach's role in fostering resilience in elite athletes. Sport, Exercise, and Performance Psychology, 8(3), 239-254. https://doi.org/10.1037/spy00 00151

Knight, L. K., Stoica, T., Fogleman, N. D., \& Depue, B. E. (2019). Convergent neural correlates of empathy and anxiety during socioemotional processing. Frontiers in Human Neuroscience, 13(March), 1-15. https://doi.org/10.3389/fnhum.2019.00094 
Machida, M., Otten, M., Magyar, T. M., Vealey, R. S., \& Ward, R. M. (2017). Examining multidimensional sport-confidence in athletes and non-athlete sport performers. Journal of Sports Sciences, 35(5), 410-418. https://doi.org/10.1080/02640414.2016.1167934

Martinent, G., \& Ferrand, C. (2007). A cluster analysis of precompetitive anxiety: Relationship with perfectionism and trait anxiety. Personality and Individual Differences, 43(7), 1676-1686. https:// doi.org/10.1016/j.paid.2007.05.005

Nakajima, M., Takano, K., \& Tanno, Y. (2019). Mindfulness relates to decreased depressive symptoms via enhancement of selfinsight. Mindfulness, 10(5), 894-902. https://doi.org/10.1007/ s12671-018-1049-2

Pena, M., \& Losada, L. (2017). Test anxiety in Spanish adolescents: Examining the role of emotional attention, and ruminative selffocus and regulation. Frontiers in Psychology, 8(AUG), 1-7. https://doi.org/10.3389/fpsyg.2017.01423

Pijpers, J. R., Oudejans, R. R. D., \& Bakker, F. C. (2005). Anxietyinduced changes in movement behaviour during the execution of a complex whole-body task. Quarterly Journal of Experimental Psychology Section A: Human Experimental Psychology, 58(3), 421-445. https://doi.org/10.1080/02724980343000945

Pines, M., \& Rutter, M. (1984). Resilient children.

Rhodes, R. E., \& Smith, N. E. I. (2006). Personality correlates of physical activity: A review and meta-analysis. British Journal of Sports Medicine, 40(12), 958-965. https://doi.org/10.1136/bjsm.2006. 028860

Rice, S. M., Gwyther, K., Santesteban-Echarri, O., Baron, D., Gorczynski, P., Gouttebarge, V., Reardon, C. L., Hitchcock, M. E., Hainline, B., \& Purcell, R. (2019). Determinants of anxiety in elite athletes: A systematic review and meta-analysis. British Journal of Sports Medicine, 53(11), 722-730. https://doi.org/10.1136/ bjsports-2019-100620

Richardson, G. E., Neiger, B. L., Jensen, S., \& Kumpfer, K. L. (1990). The resiliency model. Health Education, 21(6), 33-39.

Rodriguez-Ayllon, M., Cadenas-Sánchez, C., Estévez-López, F., Muñoz, N. E., Mora-Gonzalez, J., Migueles, J. H., Molina-García, P., Henriksson, H., Mena-Molina, A., Martínez-Vizcaíno, V., Catena, A., Löf, M., Erickson, K. I., Lubans, D. R., Ortega, F. B., \& Esteban-Cornejo, I. (2019). Role of physical activity and sedentary behavior in the mental health of preschoolers, children and adolescents: a systematic review and meta-analysis. Sports Medicine, 1383-1410. https://doi.org/10.1007/s40279-019-01099-5

Sallis, J., Prochska, J., \& Taylor, W. (2000). Parental rules for physical activity. Epidemiology, 1, 963-975.

Sarkar, M., \& Fletcher, D. (2014). Psychological resilience in sport performers: A review of stressors and protective factors. Journal of Sports Sciences, 32(15), 1419-1434. https://doi.org/10.1080/ 02640414.2014.901551

Smith, N. C., Burwitz, L., \& Jakeman, P. (1988). Precompetitive anxiety and motor performance: A psychophysiological examination. Journal of Sports Sciences, 6(2), 115-130. https://doi.org/10. 1080/02640418808729802

Sniras, S. A., \& Uspuriene, B. A. (2018). Assessment of pre-competition emotional states of different mastery women-basketball players. Physical Education of Students, 22(3), 151. https://doi. org/10.15561/20755279.2018.0307

Wagnild, G., \& Young, H. (1993). Development and Pshycometric evaluation of a Recilience scale. Journal of Nursing Measurement, 1(2), 165-178.

Yang H. Y., Zhang L. W., Zhang L. C., Hui B. H., \& Lu, W. (2015). Cognitive dissonance and athletes' self-loss: the compensatory effect of self-affirmation. Journal of Tianjin Institute of Physical Education, (3), 249-255. https://doi.org/10.13297/j.cnki.issn1 005-0000.2015.03.013.

Zhang, S., Woodman, T., \& Roberts, R. (2018). Anxiety and fear in sport and performance. In Oxford research encyclopedia of psychology.

Zhu (1994) Anxiety Scale (CSAI-2 Questionnaire) revision of the Chinese Norm. Psychological Science (06). https://doi.org/10. 16719/j.cnki.1671-6981.1994.06.007

Zurita-Ortega, F., Chacón-Cuberos, R., Cofre-Bolados, C., Knox, E., \& Muros, J. J. (2018). Relationship of resilience, anxiety and injuries in footballers: Structural equations analysis. PLoS One, 13(11), 1-12. https://doi.org/10.1371/journal.pone.0207860

Publisher's Note Springer Nature remains neutral with regard to jurisdictional claims in published maps and institutional affiliations. 